\title{
Relation entre la production et la consommation des fruits cultivés sur le plateau d'Allada au sud du Benin
}

\author{
Christophe Cocou Tossou ${ }^{1 *}$, Anne B. FloQUeT ${ }^{2}$, Brice A. SINSIN ${ }^{3}$
}

\author{
1 INRA-Bénin, \\ BP 71, Allada, Bénin \\ chritossou@yahoo.fr \\ 2 Dép. Environ. Agric. Durable \\ CEBEDES ONG, \\ Univ. Abomey-Calavi, \\ 02 BP 331 Cotonou, Bénin \\ ${ }^{3}$ Fac. Sci. Agron. \\ Univ. Abomey-Calavi, \\ 01 BP 526, Cotonou, Bénin
}

* Correspondance et tirés à part

Reçu le 25 juin 2010

Accepté le 16 mai 2011

Fruits, 2012, vol. 67, p. 3-12 (C) 2011 Cirad/EDP Sciences All rights reserved DOI: 10.1051/fruits/2011061 www.fruits-journal.org

RESUMEN ESPAÑOL, p. 12

\section{Relationship between production and consumption of fruit grown on the Allada plateau in southern Benin.}

Abstract - Introduction. In Benin, the diet of the population is deficient in vitamins and minerals; consumption of fruits and vegetables could be an effective way to prevent nutritional deficiencies and chronic disease. In this context, it was important to study the volume of fruit and vegetable consumption in Benin and to assess the relationship between availability, price and acquisition by households. We were interested in the consumption of fruits produced in a region of Benin, Allada plateau. Materials and methods. The different species of fruit grown on the Allada plateau were identified. Availability was assessed and related to their consumption based on a regional survey of a sample of 120 farm managers across four municipalities in the region. Results. The net consumption of locally grown fruits by each producing household was estimated at $31.6 \mathrm{~kg}$ per year. Among the 15 fruit species identified, the most eaten were found to be mango (13.3 kg per person per year, $42 \%)$, banana (7.6 kg per person per year, $24 \%)$, mandarin (11\%), orange (8\%) and avocado (7\%). No significant correlation was found between consumption and production of fruit. However, a very strong correlation was demonstrated between the production and the amount of fruit sold, and a significant negative correlation was found between the amount of fruit products and their selling price. The balance between production and consumption showed a positive margin for the orange, pineapple, tangelo and papaya, which means that these products are primarily commercial and only a residual share of these crops is reserved for consumption. Most other fruit crops consumed by households of Allada plateau must be imported. Discussion. On the Allada plateau, despite high production of certain fruits, their consumption by farm households is low and little diversified with respect to their availability; it is well below the standards recommended by the WHO. It will be necessary to study further the determinants of such a bias to guide public policy and the choice of producers and consumers better to ensure a better diet for local people.

Benin / fruit growing / farm surveys / production / on-farm consumption / marketing / marketing margins / nutritional requirements

\section{Relation entre la production et la consommation des fruits cultivés sur le plateau} d'Allada au sud du Bénin.

Résumé - Introduction. Au Bénin, le régime alimentaire des populations est déficient en vitamines et minéraux ; la consommation de fruits et légumes pourrait constituer un moyen efficace pour éviter les carences nutritionnelles et prévenir les maladies chroniques. Dans un tel contexte, il importait d'étudier le volume de consommation des fruits et légumes au Bénin et d'évaluer les liens existant entre leur disponibilité, leur prix et leur acquisition par les ménages. Nous nous sommes intéressés à l'autoconsommation des fruits produits dans une région du Bénin, le plateau d'Allada. Matériel et méthodes. Les différentes espèces fruitières cultivées sur le plateau d'Allada ont été identifiées. Leur disponibilité a été évaluée et mise en relation avec leur consommation en s'appuyant sur une enquête régionale portant sur un échantillon de 120 chefs d'exploitation agricole répartis sur quatre communes de la région. Résultats. L'autoconsommation nette des fruits cultivés localement par chacun des ménages producteurs a été évaluée à 31,6 kg par an. Parmi les 15 espèces fruitières recensées, les plus consommées se sont révélées être la mangue (13,3 kg par personne et par an, 42\%), la banane (7,6 kg par personne et par an, $18 \%$ ), l'orange (8\%) et l'avocat (7\%). Aucune corrélation significative n'a été établie entre la consommation et la production des fruits. En revanche, une très forte corrélation a été mise en évidence entre la production et la quantité de fruits commercialisée, et une corrélation négative significative a été trouvée entre la quantité de fruits produits et leur prix de vente. Le bilan entre production et consommation a fait apparaître une marge positive pour l'orange, l'ananas, le tangelo et la papaye, ce qui signifie que ces productions sont avant tout marchandes; seule une part résiduelle de ces récoltes est réservée à l'autoconsommation. La plus grande partie des autres productions fruitières consommées par les ménages du plateau d'Allada doivent être importée. Discussion. Sur le plateau d'Allada, malgré une forte production de certains fruits, leur consommation par les ménages agricoles est faible et peu diversifiée par rapport à leur disponibilité et se situe bien en deçà des normes recommandées par l'OMS. Il sera nécessaire de mieux étudier les déterminants d'une telle distorsion pour mieux guider les politiques publiques, ainsi que les choix des producteurs et des consommateurs, afin d'assurer un meilleur régime alimentaire aux populations locales.

Bénin / culture fruitière / enquête sur exploitations agricoles / production / autoconsommation / commercialisation / marge de distribution / besoin nutritionnel 


\section{Introduction}

L'alimentation est un déterminant majeur du maintien de l'organisme dans un état de santé satisfaisant et des recommandations nutritionnelles ont été formulées pour encourager une alimentation saine [1, 2]. Diverses études épidémiologiques montrent qu'une faible consommation de fruits et de légumes accroit le risque de maladies chroniques ${ }^{1}$ chez l'homme [3], et l'Afrique n'échappe pas à ce constat, bien au contraire [4]. Le rapport conjoint de l'Organisation mondiale de la Santé (OMS) et de la FAO (Food and Agriculture Organization) sur la prévention des maladies chroniques recommande ainsi une consommation d'au moins $400 \mathrm{~g}$ de fruits et légumes par personne et par jour [5]. De part leur faible charge calorique, les fruits et légumes contribuent à réduire la densité énergétique de l'alimentation [6]. L'accent est de plus en plus mis sur l'importance de la diversité des aliments et, en particulier, des fruits et légumes, ainsi que sur les synergies pouvant exister entre les constituants d'un même aliment et entre aliments. Malheureusement très peu de travaux portent sur les effets synergiques, permettant d'argumenter scientifiquement l'impact des fruits et légumes sur la santé, alors qu'une pléthore de travaux porte sur l'effet d'un principe actif sur une pathologie particulière [7]. De ce fait, la consommation de fruits et légumes est de plus en plus considérée par de nombreuses instances comme un enjeu de santé publique et fait l'objet de recommandations nutritionnelles par la FAO et l'OMS au niveau mondial [5].

L'alimentation de la population au sud du Bénin est essentiellement composée de deux produits de base riches en amidon, le maïs et le manioc, et d'un troisième, le niébé, une légumineuse riche en glucides (plus de $50 \%$ ). Les variations culinaires à partir de ces trois produits dépendent de la sauce qui accompagne les mets. De toute évidence, ce régime alimentaire est déficient en vitamines et minéraux; au Bénin, la consommation de fruits et légumes pourrait constituer un moyen efficace pour éviter les

1 Cancer, maladies cardio-vasculaires et cérébrales, diabète, obésité. carences nutritionnelles et prévenir les maladies chroniques à condition de lever certains blocages culturels. Malheureusement, les données qui pourraient orienter le choix des fruits et légumes à consommer en fonction des besoins ne sont pas disponibles.

Dans un tel contexte, il importait d'étudier le volume de consommation des fruits et légumes au Bénin et d'évaluer les liens existant entre leur disponibilité, leur prix et leur acquisition par les ménages. C'est dans cette perspective que se sont inscrits nos travaux de recherche qui se sont intéressés à l'autoconsommation des fruits produits dans une région au sud du Bénin, le plateau d'Allada.

\section{Matériel et méthodes}

\subsection{Localisation de l'étude}

Notre étude de la consommation des fruits et légumes au Bénin a été menée sur le plateau d'Allada, dans la zone guinéenne du pays (entre $6^{\circ} 25^{\prime}$ et $7^{\circ} 30^{\prime} \mathrm{N} ; 2^{\circ}$ et $2^{\circ} 30^{\prime} \mathrm{E}$ ). Ce plateau est délimité par les vallées de l'Ouémé, du Couffo et la dépression de la Lama. Il est constitué en grande partie de sols ferrugineux tropicaux, sols faiblement ferralitiques qui conviennent aux cultures vivrières, ainsi qu'aux plantations d'ananas, de palmiers à huile et d'agrumes [8]. Le régime pluviométrique de cette région est bimodal (avril-juin et septembrenovembre) avec une pluviométrie moyenne de $1200 \mathrm{~mm} \cdot \mathrm{an}^{-1}$. La température moyenne varie de $25^{\circ} \mathrm{C}$ à $29^{\circ} \mathrm{C}$ et l'humidité de l'air de $69 \%$ à $97 \%$ [9]. En 2002, la population du plateau d'Allada répartie dans les communes d'Allada, de Toffo, de Tori-Bossito et de Zê était estimée à 283900 habitants regroupés en 60124 ménages, dont 33535 ménages agricoles [10].

\subsection{Matériel et méthodes}

La distinction entre fruits, légumes, plantes à tubercules et épices est souvent arbitraire 
Tableau I.

Noms des espèces fruitières cultivées recensées auprès de 120 exploitations sur le plateau d'Allada, dans le sud du Bénin.

\begin{tabular}{lccc}
\hline Genre et espèce & Famille & Nom anglais & Nom français \\
Ananas comosus (L.) Merill & Bromeleaceae & Pineapple & Ananas \\
Anona muricata L. & Anonaceae & Soursop & Corossolier \\
Artocarpus altilis F. & Moraceae & Breadfruit tree & Arbre à pain \\
Carica papaya L. & Caricaceae & Pawpaw tree & Papayer \\
Citrus limon (L.) Burm. F. & Rutaceae & Lemon tree & Citronnier \\
Citrus maxima (Burm.) Merr. & Rutaceae & Shadek tree & Pamplemoussier \\
Citrus reticulata Blanco & Rutaceae & Mandarin tree & Mandarinier \\
Citrus sinensis (L.) Osbeck & Rutaceae & Orange tree & Oranger \\
Citrus reticulata Blanco & Rutaceae & Tangelo tree & Tangelo \\
$\times$ Citrus paradisi Macfad. & & & Mangue du Gabon \\
Irvengia gabonensis & Irvengiaceae & Crab apple tree & Manguier \\
(Aubry-Lecompte ex O'Rorke) Baill. & Sacardiaceae & Mango tree & Sapotillier \\
Mangifera indica L. & Sapotaceae & Sapodilla tree & Bananier \\
Manilkara zapota van Royen & Banana tree & Avocatier \\
Musa spp. & Musaceae & Avocado pear & Goyavier \\
Persea americana Miller & Lauraceae & Gyrtaceae &
\end{tabular}

et floue. En effet, selon le degré de maturité de leurs fruits, de nombreuses espèces se consomment fraîches comme fruits ou cuites comme légumes. Par ailleurs, tous les produits végétaux consommés essentiellement pour leur goût rafrâichissant ou aromatique sont classés comme fruits. Il s'agit en particulier de fruits à pulpe juteuse [11]. Les espèces cultivées que nous avons étudiées se sont révélées être de type arboricole, arbustif et herbacé (tableau I).

Un inventaire taxonomique exhaustif des espèces fruitières cultivées a été fait selon la méthode de Gounot [12] auprès d'un échantillon aléatoire de 120 chefs d'exploitation agricole de quatre communes (Allada, Toffo, Tori Bossito et Zê) du plateau d'Allada et en s'appuyant sur La flore $d u$ Bénin [13]. Pour la consommation des fruits, un diagnostic rapide a été effectué auprès du même échantillon d'exploitants, en s'appuyant sur les travaux de Kumar [14] et sur ceux de Beaudoux et al. [15].
Le choix des exploitations agricoles échantillonnées s'est appuyé sur deux critères principaux :

(i) exploitations présentant une surface supérieure ou égale à 0,5 ha ;

(ii) exploitations pratiquant des cultures fruitières depuis au moins 6 ans. En effet, une étude préalable que nous avions réalisée en 2001 avait conclu que 65 \% des producteurs de fruits disposaient d'une superficie supérieure ou égale à 0,5 ha [16]. Par ailleurs, un verger âgé d'au moins 6 ans est normalement en mesure de produire correctement lorsqu'il s'agit de plants greffés ou suffisamment robustes pour résister aux sécheresses ordinaires.

\subsection{Rodage de la méthode}

Tout d'abord, l'objectif de l'étude a été expliqué aux exploitants sélectionnés lors d'une première visite sur le terrain. Une 
pré-enquête auprès de six exploitants nous a ensuite permis de tester et de réviser un questionnaire adapté à notre objectif. La version définitive de ce document a été utilisée lors des visites ultérieures.

\subsection{Paramètres mesurés}

Les données recueillies ont été les suivantes :

- la quantité totale de fruits produits pour une espèce $i$ : 'Qi',

- la quantité brute de ces fruits consommée par les ménages: 'Qai',

- la quantité nette de ces fruits consommée par les ménages : 'qai',

- la quantité de fruits commercialisés 'Qc'.

La quantité nette 'qai' a été calculée en multipliant la quantité brute 'Qai' par le taux d'utilisation pour la consommation 'Tc' de chacun des fruits identifiés par notre enquête.

Le taux d'utilisation pour la consommation 'Tc' correspond au taux de partie comestible du fruit: après avoir pesé les fruits entiers, nous les avons pelés ou épluchés afin de disposer de la partie comestible. La partie comestible et la partie rejetée ont été pesées séparément. Par suite, $p$ étant le poids de la partie comestible et $P$ étant le poids du fruit entier, $\left[\mathrm{Tc}_{(\%)}=(p / P) \times 100\right]$.

Pour chacune des espèces $i$, le bilan 'B' a été obtenu par la différence entre les quantités de fruits produites 'Qi' et celles autoconsommées 'Qai', soit [Bi = Qi - Qai].

\subsection{Analyses}

Les données ont été analysées à l'aide du logiciel Minitab.

La consommation annuelle par individu a été estimée en divisant la production nette consommée par ménage 'qai' par la taille moyenne de chaque ménage.

Des corrélations (Pearson, $P<0,05$ ) ont été établies entre les éléments suivants :

- la consommation brute 'Qai' et la production totale de fruits 'Qi',
- la production commercialisée 'Qc' et la production totale ' $\mathrm{Qi}$ ',

- la quantité commercialisée 'Qc' et le prix de vente des fruits.

La quantité nette consommée 'qai' a été comparée à la norme OMS/FAO (2005) de $146 \mathrm{~kg}$ par personne et par an (soit $400 \mathrm{~g}$ par personne et par an) afin de déterminer si la production constatée suffisait à la consommation des ménages de la zone étudiée.

Les préférences de consommation des fruits chez les personnes interrogées ont été évaluées lors de l'enquête. Par extrapolation, elles ont permis d'estimer les besoins de consommation brute 'Qai' pour la population échantillon (chefs d'exploitation et leurs familles).

Ces besoins ont été comparés à la production brute 'Qi' de la population échantillon, ce qui a permis d'évaluer l'écart entre production et consommation, à partir des préférences de la population enquêtée.

\section{Résultats}

\subsection{Production fruitière sur le plateau d'Allada}

Parmi les 15 productions fruitières recensées au cours de notre enquête (tableau I), l'ananas s'est révélé majoritaire avec une production totale de $1265 \mathrm{t}^{-\mathrm{an}^{-1}}$ pour l'ensemble des exploitants enquêtés. Au sein des espèces ligneuses, l'oranger a dominé avec une production estimée à $996 \mathrm{t}^{-a n^{-1}}$ pour l'échantillon considéré. Cette production a été suivie par le mandarinier avec $126 \mathrm{t} \cdot \mathrm{an}^{-1}$. Les autres espèces recensées (avocatier, bananier, citronnier, corossollier, goyavier, manguier, papayer, pamplemoussier, sapotillier, Artocarpus altilis et Irvingia gabonensis) ne sont que faiblement cultivées (figure 1).

\subsection{Destination des cultures fruitières sur le plateau d'Allada}

D'après notre enquête, la quasi totalité des fruits $(99,40 \%)$ de la production est destinée 
à la vente ; le reste, à l'auto consommation (figure 1). Les revenus imputables à la production ont été estimés à $170 \mathrm{M} \mathrm{FCFA}^{2}$ pour l'ensemble des 120 chefs d'exploitation interrogés, générant un revenu brut moyen de 1,4 M FCFA (soit environ 2900 US\$) par producteur et par an, hors consommation domestique. Par extrapolation cette recette a été estimée à 47 Md FCFA (soit environ 98 M US\$) pour l'ensemble du plateau d'Allada.

\subsection{Les résultats de consommation}

La consommation nette (partie comestible) annuelle de l'ensemble des fruits cultivés sur le plateau d'Allada a été évaluée à 31,6 kg par ménage agricole enquêté et à, en moyenne, 4,5 kg par individu, sachant qu'il y a en moyenne sept personnes par ménage enquêté (tableau II).

Les fruits les plus consommés (consommation nette de fruits) par les ménages producteurs sont la mangue (13,3 kg soit $42 \%)$, la banane $(7,6 \mathrm{~kg}$, soit $24 \%)$, la mandarine (11\%), l'orange (8\%) et l'avocat (7\%) (figure 2).

\subsection{Liens entre production, consommation, commercialisation et prix}

Après enquête auprès de 120 ménages producteurs de fruits sur le plateau d'Allada, aucune corrélation n'a pu être mise en évidence entre la production et la consommation des fruits $\left(r=0,06, P_{0,05}=0,27\right)$. En revanche, il est apparu une très forte corrélation (voisine de 1) entre la production et la quantité de fruits commercialisée, et une corrélation négative significative $(r=0,1953$, $\left.P_{0,05}=0,007\right)$ entre la quantité de fruits commercialisée et leur prix de vente; il existe donc un lien entre production, commercialisation et prix ; ce lien n'existant pas entre production et consommation, la production de fruits sur le plateau d'Allada se révèle être plus une production marchande qu'une production vivrière.

$21 \mathrm{FCFA}=0.00152449 \mathrm{EUR}$;

1 FCFA $=0.00207921$ US\$.

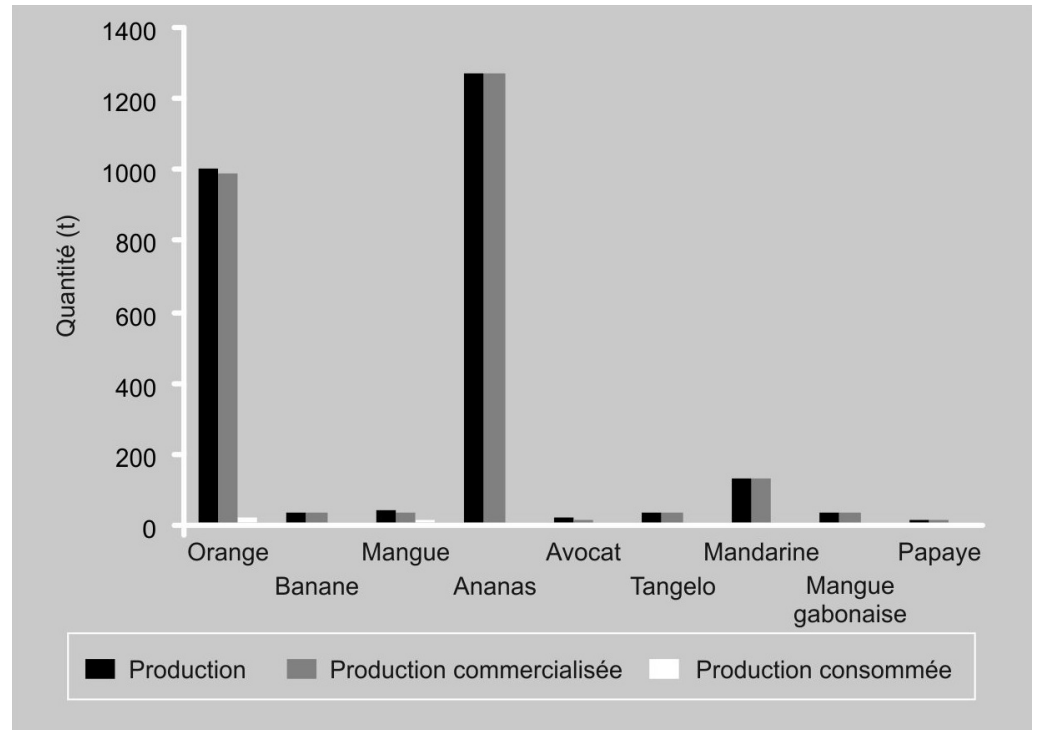

\subsection{Bilan des productions fruitières du plateau d'Allada}

Le bilan financier obtenu à partir de la commercialisation des fruits produits sur le plateau d'Allada se révèle être positif pour l'orange, l'ananas, le tangelo et la papaye qui représentent donc des cultures de rente pour les producteurs. Tous les autres fruits (avocat, banane, citron, corossol, fruit à pain, goyave, mangue, mangue gabonaise, mandarine, sapotille) doivent être majoritairement importés (figure 3).

\section{Discussion}

Notre étude a permis pour la première fois d'obtenir des données chiffrées portant sur l'importance de l'autoconsommation de fruits et sa liaison avec la production dans quatre communes du plateau d'Allada dans le sud du Bénin. Très complémentaire des méthodes de recherche conventionnelles, le diagnostic participatif utilisé s'est révélé être adapté à l'obtention rapide, avec peu de moyens, d'informations pertinentes sur le bilan financier des productions fruitières dans cette région. Les résultats permettent d'appréhender les grandes lignes d'une situation agricole dans un milieu rural peu documenté et d'orienter des investigations
Figure 1.

Répartition de la production fruitière sur le plateau d'Allada (Bénin) selon les données fournies par un échantillonnage de 120 exploitants. 
Tableau II.

Consommation annuelle de fruits produits par 120 ménages exploitants, enquêtés au Bénin (plateau d'Allada).

$\begin{array}{lcccc}\text { Fruit } & \begin{array}{c}\text { Consommation brute de fruits } \\ \text { (kg par personne) }\end{array} & \begin{array}{c}\text { Valeurs relatives } \\ (\%)\end{array} & \begin{array}{c}\text { Rendement } \\ \text { des fruits } \\ (\%)\end{array} & \begin{array}{c}\text { Consommation nette } \\ \text { de fruits } \\ (\mathrm{kg})\end{array} \\ \text { Mangue } & 30,20 & 49,59 & 0,44 & 13,29 \\ \text { Banane } & 10,17 & 16,70 & 0,75 & 7,63 \\ \text { Mandarine } & 5,77 & 9,47 & 0,60 & 3,46 \\ \text { Orange } & 6,35 & 10,43 & 0,40 & 2,54 \\ \text { Avocat } & 3,87 & 6,35 & 0,60 & 2,32 \\ \text { Mangue gabonaise } & 3,25 & 5,34 & 0,50 & 1,63 \\ \text { Fruit à pain } & 0,67 & 1,10 & 0,60 & 0,40 \\ \text { Corossol } & 0,19 & 0,31 & 0,75 & 0,14 \\ \text { Papaye } & 0,13 & 0,21 & 0,60 & 0,08 \\ \text { Goyave } & 0,20 & 0,33 & 0,30 & 0,06 \\ \text { Sapotille } & 0,05 & 0,08 & 0,50 & 0,03 \\ \text { Citron } & 0,05 & 0,08 & 0,20 & - \\ \text { Ananas } & 0 & - & - & 0,46 \\ \text { Tangelo } & 0 & 100,00 & 0,60 & - \\ \text { Total } & 60,90 & & - & 31,58\end{array}$

Figure 2.

Consommation de fruits par ménage agricole sur le plateau d'Allada (Bénin) selon les données fournies par un échantillonnage de 120 exploitants.

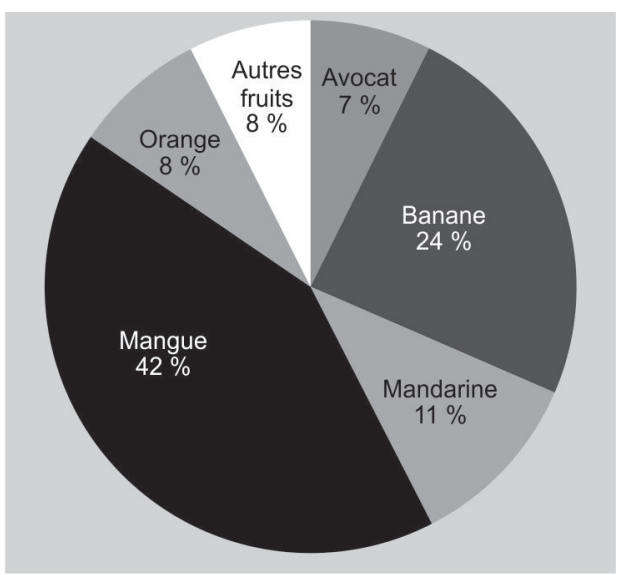

à venir afin d'en préciser les diverses composantes [17-20]. Mais nos travaux n'ont abordé que quelques aspects spécifiques de cet état de fait et n'ont fourni que des informations partielles sur des situations complexes [21].

Si l'inventaire réalisé a permis de dénombrer les espèces présentes dans les exploi- tations agricoles échantillonnées, il nous est difficile de vérifier les quantités de fruits produites, vendues et autoconsommées. Dès lors, il serait intéressant de mener une étude similaire sur un échantillon de chefs d'exploitation plus important afin de mieux couvrir la zone d'étude et d'élargir l'enquête à d'autres régions pour disposer de bases fiables, aptes à aider à la mise en œuvre d'un réel programme de développement fruitier au Bénin. Par ailleurs, un suivi du régime alimentaire des ménages se révèlerait nécessaire pour mieux préciser les données de consommation qui n'ont été que déclaratives lors de notre étude.

Malgré ces limites, nos résultats suggèrent que, dans l'ensemble des communes d'Allada, Toffo, Tori Bossito et de Zê au sud du Bénin, les cultures fruitières cultivées sont aptes à constituer d'importantes sources de revenus. Mais l'autoconsommation de ces fruits par les ménages agricoles est faible et peu diversifiée par rapport aux normes de l'OMS/FAO [5] et à la disponibilité de ces produits. 


\subsection{Niveau de consommation des fruits}

Au Bénin, la forte consommation de mangue constatée est due au double rôle du manguier en tant qu'arbre fruitier, mais aussi en tant que production vivrière. Arrivant à maturité en fin de saison sèche et en début de saison humide, les mangues constituent un apport nutritif fondamental dans l'alimentation des populations rurales des zones d'Afrique occidentale [22]. La consommation de fruits dans les ménages producteurs sur le plateau d'Allada, estimée à 4,5 kg par personne et par an, soit environ $12 \mathrm{~g}$ par jour, est de loin inférieure à la moyenne mondiale et africaine. À titre comparatif et selon les données de la FAO à l'échelle mondiale [6], la consommation apparente de fruits serait relativement élevée en Amérique latine (271 g par jour), en Europe (212 g par jour) et dans le MoyenOrient (204 g par jour), alors qu'elle serait beaucoup plus basse en Asie (85 g par jour) et en Afrique (95 g par jour). Un exemple est donné par un pays comme le Vietnam où la consommation annuelle de fruits a été estimée à 4 Mt en 2001, soit à 137 g par personne et par jour; pour ce pays, la progression annuelle de la production fruitière a été estimée à 2,3\% par an sur les quinze dernières années; le marché intérieur permet d'écouler $93 \%$ de cette production, cela surtout vers les centres urbains qui, avec moins d'un quart de la population, représenteraient près de la moitié du marché domestique des fruits. [23]. Un tel modèle qui met l'accent sur une production à destination du marché intérieur pourrait être riche d'enseignements pour des plans d'actions à mettre en œuvre en Afrique et plus particulièrement au Bénin où les marchés visés sont plutôt le marché européen et éventuellement le marché régional.

En Afrique francophone, il existe une grande diversité de situations selon la position géographique des pays, leurs traditions culturelles et leur situation économique. De nombreuses populations consomment beaucoup moins que $400 \mathrm{~g}$ de fruits et légumes par personne et par jour. Dans les pays de forêts humides (Cameroun, Gabon,

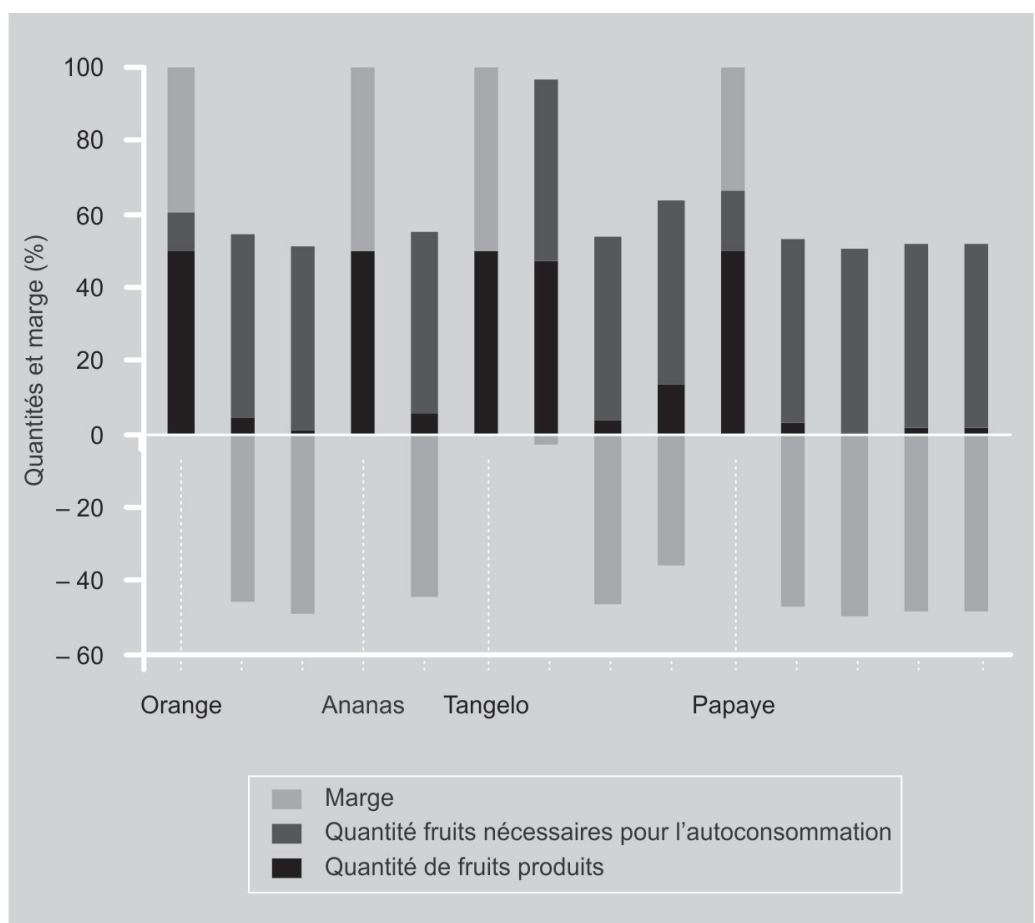

Guinée, Rwanda et Burundi), la banane et les plantains sont des fruits très consommés, ce qui explique des niveaux de consommation de fruits relativement élevés. En revanche, dans les pays sahéliens (BurkinaFaso, Tchad, Mali et Mauritanie) la situation est très critique. [4]. Le Bénin se classe dans cette dernière catégorie.

\subsection{Liens entre production, consommation, commercialisation et prix}

Notre étude a fait ressortir que le niveau d'autoconsommation des fruits ne dépendait pas de leur disponibilité et que, donc, d'autres facteurs entraient en jeu pour influencer la consommation des fruits par les ménages producteurs; en particulier une priorité serait donnée à la commercialisation des productions. Ces résultats confirment ceux de Sodjinou et al. selon lesquels la cause fondamentale d'une faible consommation de fruits et légumes serait liée une information insuffisante de la population sur la valeur nutritionnelle de ces aliments et leur impact sur la santé [24]. Ces
Figure 3.

Bilan entre la production et les besoins d'autoconsommation de fruits sur le plateau d'Allada effectué à partir de données fournies par enquête auprès de 120 exploitants d'espèces fruitières. 
auteurs concluent que l'alimentation pauvre en fruits et légumes des adultes de la ville de Cotonou est loin d'assurer une bonne adéquation entre micro nutriments et prévention satisfaisante des maladies cardio vasculaires. Cette situation s'apparenterait à celle des ménages producteurs de fruits des communes du plateau d'Allada.

Les corrélations positives observées entre production, quantité commercialisée et prix de vente des fruits indiquent quils sont avant tout sources de revenus pour les ménages producteurs ; par suite la partie autoconsommée correspondrait aux excédents de production ou aux invendus. Ce constat serait un handicap dans le contexte d'une tentative d'accroissement de la consommation des fruits dans la région, pour améliorer le statut nutritionnel de ses populations.

\section{Conclusion}

Sur le plateau d'Allada au Bénin, les fruits cultivés sont principalement destinés à la vente et peu utilisés pour l'alimentation des ménages producteurs. Il en résulte un déficit de consommation par rapport aux recommendations dispensées par l'OMS. Contrairement à toute logique économique classique [25], il n'existe pas de lien établi entre la production et l'autoconsommation des fruits. D'autres éléments, probablement de multiples origines, entreraient donc en jeu qui freineraient la consommation adéquate de fruits et légumes dans cette région. Pour y faire face, il apparait nécessaire de décloisonner les secteurs d'intervention [26]. En particulier, les domaines de la santé, de l'horticulture et de l'éducation devraient œuvrer ensemble, tout comme ceux des transports et de l'environnement. Une sensibilisation des enfants à l'importance des fruits et légumes pour la santé serait primordiale dans certains pays où les produits industriels à forte charge calorique séduisent les nouveaux consommateurs.

Notre étude réalisée au Bénin, dans une région productrice de fruits, devra s'élargir au niveau national et prendre en compte la saisonnalité de production des fruits au niveau des divers bassins, ainsi que leur dis- ponibilité, en liaison avec le statut nutritionnel et sanitaire des populations rurales et urbaines à l'échelle du pays. Nous espérons en outre que nos travaux pourront aider à construire une méthodologie robuste afin de conduire des études similaires dans d'autres situations géographiques, et ainsi gagner en généricité.

\section{Remerciements}

Les auteurs remercient le Dr. Jacky Ganry pour ses conseils lors de la rédaction du manuscrit.

\section{Références}

[1] Martin A., Apports nutritionnels conseillés pour la population française, AFSSACNERNA-CNRS, Ed. Tec. \& Doc. Lavoisier, Paris, France, 2001.

[2] Anon., Pour une politique nutritionnelle de santé publique en France. Enjeux et propositions, Min. Empl. Solidar., Haut Com. Santé Publique (HCSP), Paris, France, 2000.

[3] Gember M., Boutron-Ruault M.-C., Hercberg S., Riboli E., Scalbert A., Siess M.-H., Actualités en cancérologie : fruits, légumes et cancers. Une synthèse du réseau Nacre, Bull. Cancer 89 (3) (2002) 293-312.

[4] Ganry J., Current status of fruits and vegetables production and consumption in francophone african countries. Potential Impact on Health, Acta Hortic. 841 (2009) 249-255.

[5] Anon., Fruit and vegetables for health. Rep. Jt. FAO/OMS/OMS Workshop, 1-3 Sept. 2004, Koba, Japan, World Health Organ., Geneva, Switz., 2005.

[6] Combris P., Amiot M.-J., Caillavet F., Cause M., Dallongeville J., Padilla M., Renard C., Soler L.-G., Les fruits et légumes dans l'alimentation. Enjeux et déterminants de la consommation. Expertise scientifique collective Inra, Quae, Coll. Expertises collectives, Versailles, France, 2008.

[7] Ganry J., Effects of the fruit and vegetables on the human health, Fruits 62 (6) (2007) 343-344.

[8] Bremer F., Busacker D., Diallo A., Fehlberg H., Meyer C., Monigatti W., Spiegel K.-H., 
Les possibilités de promotion des petites exploitations agricoles dans la province Atlantique (Bénin) : étude préparée pour le projet Carder Atlantique (GTZ), Techn. Univ. Berlin. [Fachbereich Int. Agrarentwickl. (FIA)], Margraf, Aichtal Germany, 1986, $195 \mathrm{p}$.

[9] Assogbadjo A.E., Sinsin B., van Damme P., Caractères morphologiques et production des capsules de baobab (Adansonia digitata) au Bénin, Fruits 60 (5) (2005) 327-340.

[10] Anon., Étude sur les conditions de vie des ménages ruraux au Bénin, MAEP/ DANIDA/ PNUD, Bénin, 2001, 84 p.

[11] Bärtels A., Guide des plantes tropicales. Plantes ornementales, plantes utiles, fruits exotiques, Ed. Ulmer, Allemagne, 1994, $384 \mathrm{p}$.

[12] Gounot N., Méthodes d'étude quantitative de la végétation, Masson, Paris, France, 1969, $314 \mathrm{p}$.

[13] De Souza S., Flore du Bénin, Tome 3, Ed. Presse Notre Dame, Cotonou, Bénin, 1988, $424 \mathrm{p}$.

[14] Kumar K., An overview of rapid appraisal methods in developmental settings, Agency Int. Dev., Wash., D.C., U.S.A., 1990.

[15] Beaudoux E., Grombrugghe G., Douxchamps F., Gueneau M.-J., Nieuwkerk M., Cheminements d'une action de développement. De l'identification à l'évaluation, L'Harmattan, 1992, Paris, France, 206 p.

[16] Tossou C.C., Impact de la culture de l'ananas sur l'environnement dans le département de l'Atlantique, in : Agbo B.P., Isidore T.I., Adjanohoun A., Sagbohan J., Ganglo J., Bankolé C., Igué K., Matthess A., Recherche agricole pour le développement, Actes Atelier Sci., Niaouli 12-13 décembre 2001, Ed. INRA Bénin, Bénin, 2003, 538555.
[17] Chambers R., Rural appraisal: rapid, relaxed, and participatory, Inst. Dev. Stud., Univ. Sussex, U.K., 1992.

[18] Dunn T., Rapid rural appraisal: a description of the methodology and its application in teaching and research at Charles Sturt University, Cent. Rural Res., Wagga Wagga, Aust., 1994.

[19] Anon., Rapid rural appraisal, in: Proc. 1985 Int. Conf., Khon Kaen Univ., Thailand, 1985.

[20] Kumar K., Casley D.J., The collection, analysis, and use of monitoring and evaluation data, Johns Hopkins Univ. Press, Baltimore, M.D., U.S.A., 1993.

[21] Gausset Q., Ranking local tree needs and priorities through an interdisciplinary action research approach, J. Transdiscipl. Environ. Stud. 3 (1) (2004) 1-17.

[22] Vayssières J.-F., Korie S., Coulibaly O., Temple L., Boueyi P.S., Le manguier dans le nord et le centre du Bénin: inventaire des variétés, évaluation des rendements, stades de contamination et pertes dues au mouches des fruits (Diptera: Tephritidae), Fruits 63 (6) (2008) 335-348.

[23] Figuié M., Bricas N., Amélioration des productions fruitières au Vietnam, in: Atelier Impact de 10 années de coopération française sur l'amélioration des productions fruitières au Vietnam, 19-20 mai 2004, Long Dinh, Vietnam, 2004

[24] Sodjinou R., Delisle H., Fayomi B., Agueh V., L'alimentation des adultes de Cotonou estelle adéquate pour prévenir des maladies cardivasculaires ? Univ. Montréal, Inst. Rég. Santé Publique (Ouidah), Inst. Sci. Bioméd. Appl., Commun., Cotonou, Bénin, 2007.

[25] Roux P., Économie agricole. Les fondements de l'économie, Tec. \& Doc. Lavoisier, Paris, France, 1986, $25 \mathrm{p}$.

[26] Ganry J., Santé : l'Afrique doit augmenter sa consommation de fruits et légumes, Cirad, Lett. Inf., Montpellier, France, 2008, 1 p. 


\section{Relación entre la producción y el consumo de frutos cultivados en la meseta} de Allada, al sur de Benín.

Resumen - Introducción. En Benín, el régimen alimentario de las poblaciones carece de vitaminas y minerales, de modo que el consumo de frutas y verduras podría constituir un medio eficaz para evitar insuficiencias nutricionales y prevenir enfermedades crónicas. En este contexto, fue importante estudiar el volumen de consumo de frutas y verduras en Benín y evaluar la vinculación existente entre su disponibilidad, su precio y su adquisición en los hogares. Nos centramos en el consumo propio de las frutas producidas en una región de Benín, la meseta de Allada. Material y métodos. Se identificaron las diferentes especies frutales cultivadas en la meseta de Allada. Se evaluó su disponibilidad y se relacionó con su consumo, mediante un sondeo regional, basado en un muestreo de 120 jefes de explotación agrícola, repartidos en cuatro comunas de la región. Resultados. Se evaluó en 31,6 kg por año, el consumo propio neto de las frutas cultivadas localmente por cada hogar productor. Entre las 15 especies frutales inventariadas, las más consumidas resultaron ser el mango $(13,3 \mathrm{~kg}$ por persona y por año, $42 \%)$, el banano $(7,6 \mathrm{~kg}$ por persona y por año, $18 \%)$, la naranja ( $8 \%$ y el aguacate $(7 \%)$. No se estableció ninguna correlación significativa entre el consumo y la producción de frutas. En cambio, se puso de manifiesto una correlación muy fuerte entre la producción y la cantidad de frutas comercializadas, del mismo modo que se encontró una importante correlación negativa entre la cantidad de frutas producidas y su precio de venta. El balance entre la producción y el consumo mostró un margen positivo para la naranja, la piña, el tangelo y la papaya, lo que significa que esas producciones son, ante todo, comerciales; únicamente una parte residual de esas cosechas está reservada al consumo propio. La mayor parte del resto de las producciones frutales consumidas por los hogares de la meseta de Allada es de importación. Discusión. A pesar de una fuerte producción de ciertos frutos en la meseta de Allada, su consumo en los hogares agrícolas es flojo y poco variado en cuanto a su disponibilidad y se sitúa muy por debajo de las normas recomendadas por la OMS. Será necesario estudiar mejor los determinantes de una semejante distorsión, de modo a guiar mejor las políticas públicas, así como la elección de los productores y consumidores, con el fin de garantizar un mejor régimen alimenticio para las poblaciones locales.

Benin / fruticultura / encuestas sobre explotaciones / producción / autoconsumo / mercadeo / margen de distribución/ necesidades de nutrientes 